\title{
Upper bounds for the number of factors for a class of polynomials with rational coefficients
}

\author{
by \\ Nicolae Ciprian Bonciocat (Bucureşti)
}

1. Introduction. Some results related to Hilbert's irreducibility theorem have been provided in [1]-[4]. In [1] it is shown that for any relatively prime polynomials $f(X), g(X) \in \mathbb{Q}[X]$ with $\operatorname{deg} f<\operatorname{deg} g$, the polynomial $f(X)+p g(X)$ is irreducible over $\mathbb{Q}$ for all but finitely many prime numbers $p$. In [2] this result has been improved by providing an explicit lower bound $b$ depending on $f$ and $g$, such that for all primes $p>b$, the polynomial $f(X)+p g(X)$ is irreducible over $\mathbb{Q}$.

Let now $f, g \in \mathbb{Q}[X]$ be relatively prime polynomials with $\operatorname{deg} f \leq \operatorname{deg} g$.

In the present paper we adapt the method in [2] in order to provide explicit upper bounds for the number of factors over $\mathbb{Q}$ of the polynomials $n_{1} f(X)+n_{2} g(X)$, where $n_{1}$ and $n_{2}$ are nonzero integers with absolute value of $n_{2} / n_{1}$ greater than an explicit lower bound $b$. Here and henceforth, by the number of factors of a polynomial $f$ we shall understand the number of irreducible factors of $f$ counted with multiplicities.

We treat separately the cases $\operatorname{deg} f<\operatorname{deg} g$ and $\operatorname{deg} f=\operatorname{deg} g$.

In the first case we prove that for any nonzero integers $n_{1}$ and $n_{2}$ with absolute value of $n_{2} / n_{1}$ greater than an explicit lower bound $b$ depending on $f$ and $g$, the number of factors over $\mathbb{Q}$ of the polynomial $n_{1} f(X)+n_{2} g(X)$ cannot exceed the total number of prime factors of $n_{2}$ counting multiplicities. We actually prove a slightly more general version of this result, in which the lower bound $b$ and the upper bound for the number of factors depend on a suitable divisor of $n_{2}$. As a corollary we find an improved form of the irreducibility criterion given in [2, Th. 1]. Sharper bounds are then obtained for polynomials with integral coefficients. We finally consider the case when the polynomial $n_{1} f(X)+n_{2} g(X)$ has no rational roots.

2000 Mathematics Subject Classification: Primary 11C08.

This work was partially supported by the CERES Program of the Romanian Ministry of Education, Youth and Research, contract no. 39/2002 and by the EURROMMAT program ICA1-CT-2000-70022 of the European Commission. 
Similar results are provided in the case $\operatorname{deg} f=\operatorname{deg} g$.

For any polynomial $f \in \mathbb{Q}[X]$ of degree $k$, we write $f(X)$ uniquely in the reduced form

$$
f(X)=\frac{a_{0}+a_{1} X+\ldots+a_{k} X^{k}}{q}
$$

where $q, a_{0}, \ldots, a_{k} \in \mathbb{Z}, a_{k} \neq 0, q \geq 1, q$ as small as possible. Then for this reduced form we set

$$
H(f)=\max \left\{\left|a_{0}\right|,\left|a_{1}\right|, \ldots,\left|a_{k}\right|, q\right\}, \quad M(f)=\max \left\{\left|a_{0}\right|,\left|a_{1}\right|, \ldots,\left|a_{k}\right|\right\} .
$$

For any integer $n$ with $|n|>1$, we denote by $\Omega(n)$ the total number of prime factors of $n$ counting multiplicities.

In the case $\operatorname{deg} f<\operatorname{deg} g$ we prove the following results:

TheOREM 1. Let $f(X), g(X) \in \mathbb{Q}[X]$ be relatively prime polynomials with $k=\operatorname{deg} f<\operatorname{deg} g=m$. Then for any nonzero integers $n_{1}, n_{2}$ and any positive divisor $d$ of $n_{2}$ such that

$$
\left|\frac{n_{2}}{n_{1}}\right|>\left(2+\frac{1}{2^{k+1} d^{m}}\right)^{k+1} d^{m} H(f)^{m} H(g)^{m+1},
$$

the polynomial $n_{1} f(X)+n_{2} g(X)$ has at most $\Omega\left(n_{2} / d\right)$ factors over $\mathbb{Q}$.

Corollary 1 . For any relatively prime polynomials $f(X), g(X) \in \mathbb{Q}[X]$ with $k=\operatorname{deg} f<\operatorname{deg} g=m$, and any prime $p$ satisfying

$$
p>\left(2+\frac{1}{2^{k+1}}\right)^{k+1} H(f)^{m} H(g)^{m+1},
$$

the polynomial $f(X)+p g(X)$ is irreducible over $\mathbb{Q}$.

Corollary 2 (of the proof of Theorem 1). Let $f(X), g(X) \in \mathbb{Z}[X]$ be relatively prime polynomials with $k=\operatorname{deg} f<\operatorname{deg} g=m$. Then for any nonzero integers $n_{1}, n_{2}$ and any positive divisor $d$ of $n_{2}$ such that

$$
\left|\frac{n_{2}}{n_{1}}\right|>\left(2+\frac{1}{2^{k+1} d^{m} H(g)^{m+1}}\right)^{k+1} d^{m} H(f) H(g)^{m},
$$

the polynomial $n_{1} f(X)+n_{2} g(X)$ has at most $\Omega\left(n_{2} / d\right)$ nonconstant factors over $\mathbb{Z}$.

We also prove a result similar to Theorem 1 in the case when the polynomial $n_{1} f(X)+n_{2} g(X)$ has no rational roots:

Theorem 2. Let $f(X), g(X) \in \mathbb{Q}[X]$ be relatively prime polynomials with $k=\operatorname{deg} f<\operatorname{deg} g=m$. Then for any nonzero integers $n_{1}, n_{2}$ and any positive divisor $d$ of $n_{2}$ such that

$$
\left|\frac{n_{2}}{n_{1}}\right|>\left(2+\frac{1}{2^{k+1} d^{m / 2}}\right)^{k+1} d^{m / 2} H(f)^{m / 2} H(g)^{1+\max (m / 2, k)},
$$


if the polynomial $n_{1} f(X)+n_{2} g(X)$ has no rational roots, then it has at most $\Omega\left(n_{2} / d\right)$ factors over $\mathbb{Q}$.

Corollary 3 (of the proof of Theorem 2). Let $f(X), g(X) \in \mathbb{Z}[X]$ be relatively prime polynomials with $k=\operatorname{deg} f<\operatorname{deg} g=m$. Then for any nonzero integers $n_{1}, n_{2}$ and any positive divisor $d$ of $n_{2}$ such that

$$
\left|\frac{n_{2}}{n_{1}}\right|>\left(2+\frac{1}{2^{k+1} d^{m / 2} H(g)^{1+\max (m / 2, k)}}\right)^{k+1} d^{m / 2} H(f) H(g)^{\max (m / 2, k)},
$$

if the polynomial $n_{1} f(X)+n_{2} g(X)$ has no rational roots, then it has at most $\Omega\left(n_{2} / d\right)$ nonconstant factors over $\mathbb{Z}$.

In the case $\operatorname{deg} f=\operatorname{deg} g$ we prove the following results:

THEOREM 3. Let

$$
f(X)=\frac{a_{0}+a_{1} X+\ldots+a_{m} X^{m}}{q_{1}}, \quad g(X)=\frac{b_{0}+b_{1} X+\ldots+b_{m} X^{m}}{q_{2}}
$$

be relatively prime polynomials in $\mathbb{Q}[X]$ of degree $m$, written in reduced form. Let also $n_{1}, n_{2}$ be nonzero integers, $h=\left(n_{1} a_{m} q_{2}+n_{2} b_{m} q_{1}\right) / \operatorname{gcd}\left(q_{1}, q_{2}\right)$ and $d$ a positive divisor of $h$. If

$$
\left|\frac{n_{2}}{n_{1}}\right|>d^{m} H(f) H(g)\left(1+H(f) H(g)+\frac{1}{2^{m} d^{m}}\right)^{m+1},
$$

then the polynomial $n_{1} f(X)+n_{2} g(X)$ has at most $\Omega(h / d)$ factors over $\mathbb{Q}$.

Corollary 4. Let $f(X)$ and $g(X)$ be as in Theorem 3. If $n_{1}$ and $n_{2}$ are nonzero integers such that $\left|\left(n_{1} a_{m} q_{2}+n_{2} b_{m} q_{1}\right) / \operatorname{gcd}\left(q_{1}, q_{2}\right)\right|$ is a prime and

$$
\left|\frac{n_{2}}{n_{1}}\right|>H(f) H(g)\left(1+H(f) H(g)+\frac{1}{2^{m}}\right)^{m+1},
$$

then the polynomial $n_{1} f(X)+n_{2} g(X)$ is irreducible over $\mathbb{Q}$.

Corollary 5 (of the proof of Theorem 3). Let $f(X), g(X) \in \mathbb{Z}[X]$ be relatively prime polynomials of degree $m$, with leading coefficients $a_{m}$ and $b_{m}$ respectively. Let $n_{1}$ and $n_{2}$ be nonzero integers, $h=n_{1} a_{m}+n_{2} b_{m}$ and $d$ a positive divisor of $h$. If

$$
\left|\frac{n_{2}}{n_{1}}\right|>d^{m} H(f)\left(1+H(g)+\frac{1}{d^{m}[1+H(g)]^{m}}\right)^{m+1}
$$

then $n_{1} f(X)+n_{2} g(X)$ has at most $\Omega(h / d)$ nonconstant factors over $\mathbb{Z}$.

In particular, we have

COROLlary 6. Let $f(X), g(X) \in \mathbb{Z}[X]$ be relatively prime polynomials of degree $m$, with leading coefficients $a_{m}$ and $b_{m}$ respectively. If $n_{1}$ and $n_{2}$ 
are nonzero integers such that $\left|n_{1} a_{m}+n_{2} b_{m}\right|$ is a prime number and

$$
\left|\frac{n_{2}}{n_{1}}\right|>H(f)\left(1+H(g)+\frac{1}{[1+H(g)]^{m}}\right)^{m+1},
$$

then the polynomial $n_{1} f(X)+n_{2} g(X)$ is irreducible over $\mathbb{Z}$.

The proofs of these results are presented in Sections 2 and 3 below.

2. The case $\operatorname{deg} f<\operatorname{deg} g$

2.1. Proof of Theorem 1. Let

$$
f(X)=\frac{a_{0}+a_{1} X+\ldots+a_{k} X^{k}}{q_{1}} \text { and } g(X)=\frac{b_{0}+b_{1} X+\ldots+b_{m} X^{m}}{q_{2}}
$$

be two relatively prime polynomials in $\mathbb{Q}[X]$ written in reduced form, with $k=\operatorname{deg} f<\operatorname{deg} g=m$, and let also $n_{1}, n_{2}$ and $d$ be as in the statement of the theorem. Our assumption on $n_{1}, n_{2}$ and $d$ shows that $\left|n_{2}\right|>d$, so $\Omega\left(n_{2} / d\right)$ makes sense. We may obviously assume $\Omega\left(n_{2} / d\right)<m$.

We write $g(X)$ in the following form:

$$
g(X)=\frac{b_{0}+b_{1} X+\ldots+b_{m} X^{m}}{q_{2}}=\frac{\bar{b} \bar{g}(X)}{q_{2}},
$$

where $\bar{b} \in \mathbb{Z}$ and $\bar{g}(X) \in \mathbb{Z}[X], \bar{g}(X)$ primitive. Then we write

$$
n_{1} f(X)+n_{2} g(X)=\frac{a}{q} F(X)
$$

with $\operatorname{gcd}(a, q)=1$ and $F(X) \in \mathbb{Z}[X], F(X)$ primitive. Assume now that $n_{1} f(X)+n_{2} g(X)$ has more than $\Omega\left(n_{2} / d\right)$ factors. Then by Gauss's Lemma, $F(X)$ decomposes as $F(X)=F_{1}(X) \ldots F_{s}(X)$ with $\Omega\left(n_{2} / d\right)<s \leq m$ and $F_{1}(X), \ldots, F_{s}(X) \in \mathbb{Z}[X], F_{1}, \ldots, F_{s}$ primitive with $\operatorname{deg} F_{1}, \ldots, \operatorname{deg} F_{s} \geq 1$. Let $t_{1}, \ldots, t_{s} \in \mathbb{Z}$ be the leading coefficients of $F_{1}, \ldots, F_{s}$, respectively. Then one finds

$$
t_{1} \ldots t_{s}=\frac{n_{2} q b_{m}}{a q_{2}} .
$$

If $q / q_{2}=\beta / \gamma$ with $\operatorname{gcd}(\beta, \gamma)=1$, then $\beta$ divides $q_{1}$, since $q$ divides $q_{1} q_{2}$. Therefore we have $a \gamma t_{1} \ldots t_{s}=n_{2} \beta b_{m}$, and since $\Omega\left(n_{2} / d\right)<s$, at least one of the $t_{i}$ 's, say $t_{1}$, divides $d \beta b_{m}$. So we have

$$
\left|t_{1}\right| \leq d q_{1}\left|b_{m}\right| \text {. }
$$

Now we are going to estimate the resultant $R\left(\bar{g}, F_{1}\right)$. Since $\bar{g}$ and $F_{1}$ are relatively prime, $R\left(\bar{g}, F_{1}\right)$ must be a nonzero integer, so in particular

$$
\left|R\left(\bar{g}, F_{1}\right)\right| \geq 1 .
$$


If we decompose $F_{1}$, say $F_{1}(X)=t_{1}\left(X-\theta_{1}\right) \ldots\left(X-\theta_{r}\right)$, then

$$
\left|R\left(\bar{g}, F_{1}\right)\right|=\left|t_{1}\right|^{m} \prod_{1 \leq j \leq r}\left|\bar{g}\left(\theta_{j}\right)\right| .
$$

Since each root $\theta_{j}$ of $F_{1}$ is also a root of $F(X)$, we have

$$
g\left(\theta_{j}\right)=-\frac{n_{1} f\left(\theta_{j}\right)}{n_{2}}
$$

and moreover, since $f$ and $g$ are relatively prime, $f\left(\theta_{j}\right) \neq 0$ and $g\left(\theta_{j}\right) \neq 0$ for any $j \in\{1, \ldots, r\}$. The definition of $\bar{g}$ shows that

$$
\left|\bar{g}\left(\theta_{j}\right)\right| \leq q_{2}\left|g\left(\theta_{j}\right)\right| \text {. }
$$

Using now (3)-(5) we obtain

$$
\left|R\left(\bar{g}, F_{1}\right)\right| \leq\left|t_{1}\right|^{m} \frac{q_{2}^{r}\left|n_{1}\right|^{r}}{\left|n_{2}\right|^{r}} \prod_{1 \leq j \leq r}\left|f\left(\theta_{j}\right)\right| .
$$

We now proceed to find an upper bound for $\left|f\left(\theta_{j}\right)\right|$. The equality $n_{1} f\left(\theta_{j}\right)$ $+n_{2} g\left(\theta_{j}\right)=0$ implies

$$
\left(\frac{n_{1} a_{0}}{q_{1}}+\frac{n_{2} b_{0}}{q_{2}}\right)+\ldots+\left(\frac{n_{1} a_{k}}{q_{1}}+\frac{n_{2} b_{k}}{q_{2}}\right) \theta_{j}^{k}+\frac{n_{2} b_{k+1}}{q_{2}} \theta_{j}^{k+1}+\ldots+\frac{n_{2} b_{m}}{q_{2}} \theta_{j}^{m}=0,
$$

from which we deduce that

$$
\begin{aligned}
\frac{\left|n_{2} b_{m}\right|}{q_{2}}\left|\theta_{j}\right|^{m} \leq & \left(\frac{\left|n_{1} a_{0}\right|}{q_{1}}+\frac{\left|n_{2} b_{0}\right|}{q_{2}}\right)+\ldots+\left(\frac{\left|n_{1} a_{k}\right|}{q_{1}}+\frac{\left|n_{2} b_{k}\right|}{q_{2}}\right)\left|\theta_{j}\right|^{k} \\
& +\frac{\left|n_{2} b_{k+1}\right|}{q_{2}}\left|\theta_{j}\right|^{k+1}+\ldots+\frac{\left|n_{2} b_{m-1}\right|}{q_{2}}\left|\theta_{j}\right|^{m-1} \\
\leq & \left(\frac{\left|n_{1}\right| M(f)}{q_{1}}+\frac{\left|n_{2}\right| M(g)}{q_{2}}\right)\left(1+\left|\theta_{j}\right|+\ldots+\left|\theta_{j}\right|^{m-1}\right) .
\end{aligned}
$$

Therefore, either $\left|\theta_{j}\right| \leq 1$, or if not, then

$$
\frac{\left|n_{2} b_{m}\right|}{q_{2}}\left|\theta_{j}\right|^{m}<\left(\frac{\left|n_{1}\right| M(f)}{q_{1}}+\frac{\left|n_{2}\right| M(g)}{q_{2}}\right) \frac{\left|\theta_{j}\right|^{m}}{\left|\theta_{j}\right|-1},
$$

so in both cases we have

$$
\left|\theta_{j}\right|<1+\frac{1}{\left|b_{m}\right|}\left(\frac{\left|n_{1}\right| q_{2}}{\left|n_{2}\right| q_{1}} M(f)+M(g)\right) .
$$

Now, since obviously

$$
\left|f\left(\theta_{j}\right)\right| \leq \frac{M(f)}{q_{1}}\left(1+\left|\theta_{j}\right|+\ldots+\left|\theta_{j}\right|^{k}\right),
$$

inequality (7) yields

$$
\left|f\left(\theta_{j}\right)\right|<\frac{M(f)}{q_{1}} \cdot \frac{\left[1+\frac{1}{\left|b_{m}\right|}\left(\frac{\left|n_{1}\right| q_{2}}{\left|n_{2}\right| q_{1}} M(f)+M(g)\right)\right]^{k+1}-1}{\frac{1}{\left|b_{m}\right|}\left(\frac{\left|n_{1}\right| q_{2}}{\left|n_{2}\right| q_{1}} M(f)+M(g)\right)} .
$$


Instead of (8) it will be more convenient to consider

$$
\left|f\left(\theta_{j}\right)\right|<\frac{\left|b_{m}\right| M(f)}{q_{1}} \cdot \frac{\left.\left[1+\frac{1}{\left|b_{m}\right|} \mid \frac{\left|n_{1}\right| q_{2}}{\left|n_{2}\right| q_{1}} M(f)+M(g)\right)\right]^{k+1}}{\frac{\left|n_{1}\right| q_{2}}{\left|n_{2}\right| q_{1}} M(f)+M(g)} .
$$

Using now (6) and (9), we obtain

$$
\left|R\left(\bar{g}, F_{1}\right)\right|<\left|t_{1}\right|^{m}\left[\frac{\left|n_{1}\right| q_{2}}{\left|n_{2}\right| q_{1}} M(f) \frac{\left.\left|b_{m}\right|\left[1+\frac{1}{\left|b_{m}\right|} \mid \frac{\left|n_{1}\right| q_{2}}{\left|n_{2}\right| q_{1}} M(f)+M(g)\right)\right]^{k+1}}{\frac{\left|n_{1}\right| q_{2}}{\left|n_{2}\right| q_{1}} M(f)+M(g)}\right]^{r} .
$$

Since $r \geq 1$, all we need to prove is that our assumption on $n_{1}, n_{2}$ and $d$ forces

$$
\left|t_{1}\right|^{m} \cdot \frac{\left|b_{m}\right|\left[1+\frac{1}{\left|b_{m}\right|}\left(\frac{\left|n_{1}\right| q_{2}}{\left|n_{2}\right| q_{1}} M(f)+M(g)\right)\right]^{k+1}}{1+\frac{\left|n_{2}\right| q_{1} M(g)}{\left|n_{1}\right| q_{2} M(f)}}<1 .
$$

In view of (1), it is sufficient to prove that

$$
d^{m} q_{1}^{m}\left|b_{m}\right|^{m+1}\left[1+\frac{1}{\left|b_{m}\right|}\left(\frac{\left|n_{1}\right| q_{2}}{\left|n_{2}\right| q_{1}} M(f)+M(g)\right)\right]^{k+1}<1+\frac{\left|n_{2}\right| q_{1} M(g)}{\left|n_{1}\right| q_{2} M(f)},
$$

which is equivalent to

$$
d^{m} q_{1}^{m}\left|b_{m}\right|^{m-k}\left(\left|b_{m}\right|+M(g)+\frac{\left|n_{1}\right| q_{2}}{\left|n_{2}\right| q_{1}} M(f)\right)^{k+1}<1+\frac{\left|n_{2}\right| q_{1} M(g)}{\left|n_{1}\right| q_{2} M(f)} .
$$

Now since $\left|b_{m}\right| \leq M(g)$, it suffices to prove that

$$
d^{m} q_{1}^{m} M(g)^{m+1}\left(2+\frac{\left|n_{1}\right| q_{2} M(f)}{\left|n_{2}\right| q_{1} M(g)}\right)^{k+1}<\frac{\left|n_{2}\right| q_{1} M(g)}{\left|n_{1}\right| q_{2} M(f)},
$$

or equivalently,

$$
\left|\frac{n_{2}}{n_{1}}\right|>d^{m} q_{1}^{m-1} q_{2} M(f) M(g)^{m}\left(2+\frac{q_{2} M(f)}{\left|\frac{n_{2}}{n_{1}}\right| q_{1} M(g)}\right)^{k+1} .
$$

We search for a suitable $\delta$ such that if $\left|n_{2} / n_{1}\right|>\delta \cdot d^{m} q_{1}^{m-1} q_{2} M(f) M(g)^{m}$, then $\left|n_{2} / n_{1}\right|$ also satisfies (11). So it is sufficient to find a $\delta$ satisfying

$$
\delta>\left(2+\frac{1}{\delta \cdot d^{m} q_{1}^{m} M(g)^{m+1}}\right)^{k+1} .
$$

Denote $d^{m} q_{1}^{m} M(g)^{m+1}$ by $w$. A suitable candidate for $\delta$ is $\left(2+\frac{1}{2^{k+1} w}\right)^{k+1}$, since

$$
\left(2+\frac{1}{2^{k+1} w}\right)^{k+1}>\left(2+\frac{1}{\left(2+\frac{1}{2^{k+1} w}\right)^{k+1} w}\right)^{k+1} .
$$


This proves that for

$$
\left|\frac{n_{2}}{n_{1}}\right|>\left(2+\frac{1}{2^{k+1} d^{m} q_{1}^{m} M(g)^{m+1}}\right)^{k+1} d^{m} q_{1}^{m-1} q_{2} M(f) M(g)^{m}
$$

we have $\left|R\left(\bar{g}, F_{1}\right)\right|<1$, which contradicts $(2)$. The desired conclusion follows now by noting that $q_{1}^{m-1} M(f) \leq H(f)^{m}$ and $q_{2} M(g)^{m} \leq H(g)^{m+1}$. This completes the proof of the theorem.

REMARKs. 1. The inequality (12) leads to an improved version of Theorem 1. If $\left|b_{m}\right|<M(g)$ it might be useful to directly test inequality (10). Further improvements can be done, for instance, by considering the upper bound for $\left|f\left(\theta_{j}\right)\right|$ given by (8), instead of $(9)$, but they lead to more complicated assumptions on $n_{1}, n_{2}$ and $d$.

2. In [2, Th. 1], the following result has been provided:

THEOREM. For any relatively prime polynomials $f(X), g(X) \in \mathbb{Q}[X]$ with $\operatorname{deg} f<\operatorname{deg} g=m$, and any prime $p>2 m^{m} H(f)^{m+1} H(g)^{3 m}$, the polynomial $f(X)+p g(X)$ is irreducible over $\mathbb{Q}$.

For $m>1$, Corollary 1 provides a sharper bound, since

$$
\left(2+\frac{1}{2^{m}}\right)^{m} H(f)^{m} H(g)^{m+1}<2 m^{m} H(f)^{m+1} H(g)^{3 m} .
$$

3. Corollary 2 follows immediately by (12).

A result similar to Corollary 2 is the following:

Proposition 1. Let $f(X)=a_{0}+a_{1} X+\ldots+a_{k} X^{k}$ and $g(X)=b_{0}+$ $b_{1} X+\ldots+b_{m} X^{m} \in \mathbb{Z}[X]$ be two relatively prime polynomials with $k=$ $\operatorname{deg} f<\operatorname{deg} g=m$. If $n_{1}, n_{2}$ are nonzero integers and $d$ is a positive divisor of $n_{2} b_{m}$ such that

$$
\left|\frac{n_{2}}{n_{1}}\right|>\left(2+\frac{1}{2^{k+1} d^{m} H(g)^{k+1}}\right)^{k+1} d^{m} H(f) H(g)^{k},
$$

then $n_{1} f(X)+n_{2} g(X)$ has at most $\Omega\left(n_{2} b_{m} / d\right)$ nonconstant factors over $\mathbb{Z}$.

Sketch of the proof. The proof goes as that of Theorem 1, except that $q_{1}=q_{2}=1$ and instead of (1) we find $\left|t_{1}\right| \leq d$. Indeed, since we have $a t_{1} \ldots t_{s}=n_{2} b_{m}$ with $\Omega\left(n_{2} b_{m} / d\right)<s \leq m$, at least one of the $t_{i}$ 's divides $d$. Thus, instead of (10) we have to prove that

$$
\frac{d^{m}}{\left|b_{m}\right|^{k}}\left(\left|b_{m}\right|+H(g)+\frac{\left|n_{1}\right|}{\left|n_{2}\right|} H(f)\right)^{k+1}<1+\frac{\left|n_{2}\right| H(g)}{\left|n_{1}\right| H(f)} .
$$

Since $\left|b_{m}\right| \leq H(g)$ it is sufficient to prove that

$$
\left|\frac{n_{2}}{n_{1}}\right|>d^{m} H(f) H(g)^{k}\left(2+\frac{H(f)}{\left|\frac{n_{2}}{n_{1}}\right| H(g)}\right)^{k+1} .
$$


Computations as in Theorem 1 show that inequality (13) is satisfied if $\left|n_{2} / n_{1}\right|>\delta \cdot d^{m} H(f) H(g)^{k}$ with $\delta=\left(2+2^{-k-1} d^{-m} H(g)^{-k-1}\right)^{k+1}$.

2.2. Proof of Theorem 2. In this case we may obviously assume $m \geq 2$, and since the degree $r$ of the polynomial $F_{1}$ is at least 2 , it is sufficient instead of (10) to prove that

$$
d^{m} q_{1}^{m}\left|b_{m}\right|^{m-2 k}\left(\left|b_{m}\right|+M(g)+\frac{\left|n_{1}\right| q_{2}}{\left|n_{2}\right| q_{1}} M(f)\right)^{2(k+1)}<\left(1+\frac{\left|n_{2}\right| q_{1} M(g)}{\left|n_{1}\right| q_{2} M(f)}\right)^{2},
$$

or even more, that

$$
d^{m / 2} q_{1}^{m / 2}\left|b_{m}\right|^{m / 2-k}\left(\left|b_{m}\right|+M(g)+\frac{\left|n_{1}\right| q_{2}}{\left|n_{2}\right| q_{1}} M(f)\right)^{k+1}<\frac{\left|n_{2}\right| q_{1} M(g)}{\left|n_{1}\right| q_{2} M(f)} .
$$

Now, since $\left|b_{m}\right| \leq M(g)$ it suffices to prove that

$$
\left|\frac{n_{2}}{n_{1}}\right|>d^{m / 2} q_{1}^{m / 2-1} q_{2} M(f) M(g)^{m / 2}\left(2+\frac{q_{2} M(f)}{\left|\frac{n_{2}}{n_{1}}\right| q_{1} M(g)}\right)^{k+1},
$$

if $m / 2 \geq k$, and

$$
\left|\frac{n_{2}}{n_{1}}\right|>d^{m / 2} q_{1}^{m / 2-1} q_{2} M(f) M(g)^{k}\left(2+\frac{q_{2} M(f)}{\left|\frac{n_{2}}{n_{1}}\right| q_{1} M(g)}\right)^{k+1}
$$

if $m / 2<k$. So in both cases it is sufficient to prove that

$$
\left|\frac{n_{2}}{n_{1}}\right|>d^{m / 2} q_{1}^{m / 2-1} q_{2} M(f) M(g)^{\max (m / 2, k)}\left(2+\frac{q_{2} M(f)}{\left|\frac{n_{2}}{n_{1}}\right| q_{1} M(g)}\right)^{k+1} .
$$

Let $w=2^{k+1} d^{m / 2} q_{1}^{m / 2} M(g)^{1+\max (m / 2, k)}$. It is straightforward to verify that the last inequality holds for

$$
\left|\frac{n_{2}}{n_{1}}\right|>\left(2+\frac{1}{w}\right)^{k+1} d^{m / 2} q_{1}^{m / 2-1} q_{2} M(f) M(g)^{\max (m / 2, k)},
$$

which completes the proof.

Corollary 3 follows immediately from (14).

3. The case $\operatorname{deg} f=\operatorname{deg} g$

3.1. Proof of Theorem 3. We use slightly different arguments than those used in the proof of Theorem 1 . First of all, in order to see that $\Omega(h / d)$ makes sense, we have to prove that

$$
|h|>d
$$

The definition of $h$ shows that

$$
|h| \geq\left|n_{2}\right|-\left|n_{1}\right| q_{2} M(f)>0 .
$$


Indeed, if $n_{1} a_{m}$ and $n_{2} b_{m}$ have the same sign, we find $|h| \geq\left|n_{2}\right|+1$. Our assumption that

$$
\left|\frac{n_{2}}{n_{1}}\right|>d^{m} H(f) H(g)\left(1+H(f) H(g)+\frac{1}{2^{m} d^{m}}\right)^{m+1}
$$

implies $\left|n_{2}\right|>\left|n_{1}\right| q_{2} M(f)$, so we obviously have $\left|n_{2} b_{m}\right| q_{1}>\left|n_{1} a_{m}\right| q_{2}$. Thus, if $n_{1} a_{m}$ and $n_{2} b_{m}$ have opposite signs, we find

$$
|h|=\frac{\left|n_{2} b_{m}\right| q_{1}-\left|n_{1} a_{m}\right| q_{2}}{\operatorname{gcd}\left(q_{1}, q_{2}\right)} \geq\left|n_{2}\right|-\left|n_{1}\right| q_{2} M(f)>0 .
$$

Dividing now by $d$ in (16) and using again (17), we find

$$
\frac{|h|}{d}>\left|n_{1}\right| \cdot d^{m-1} H(f) H(g)\left[\left(1+H(f) H(g)+\frac{1}{2^{m} d^{m}}\right)^{m+1}-1\right]>1,
$$

which proves (15).

Now we may obviously assume $\Omega(h / d)<m$. We write again $g(X)$ in the form

$$
g(X)=\frac{b_{0}+b_{1} X+\ldots+b_{m} X^{m}}{q_{2}}=\frac{\bar{b} \bar{g}(X)}{q_{2}},
$$

where $\bar{b} \in \mathbb{Z}$ and $\bar{g}(X) \in \mathbb{Z}[X], \bar{g}(X)$ primitive. Then we write

$$
n_{1} f(X)+n_{2} g(X)=\frac{a}{q} F(X)
$$

with $\operatorname{gcd}(a, q)=1$ and $F(X) \in \mathbb{Z}[X], F(X)$ primitive.

Assume now that $n_{1} f(X)+n_{2} g(X)$ has more than $\Omega(h / d)$ factors. Then by the Gauss Lemma, $F(X)$ will decompose as $F(X)=F_{1}(X) \ldots F_{s}(X)$ with $\Omega(h / d)<s \leq m$ and $F_{1}(X), \ldots, F_{s}(X) \in \mathbb{Z}[X], F_{1}, \ldots, F_{s}$ primitive with $\operatorname{deg} F_{1}, \ldots, \operatorname{deg} F_{s} \geq 1$. Let $t_{1}, \ldots, t_{s} \in \mathbb{Z}$ be the leading coefficients of $F_{1}, \ldots, F_{s}$, respectively. Let also $\bar{q}_{1}=q_{1} / \operatorname{gcd}\left(q_{1}, q_{2}\right), \bar{q}_{2}=q_{2} / \operatorname{gcd}\left(q_{1}, q_{2}\right)$ and denote $n_{1} a_{i} \bar{q}_{2}+n_{2} b_{i} \bar{q}_{1}$ by $h_{i}$ for all $i \in\{0, \ldots, m-1\}$. Since

$$
\frac{h_{0}+h_{1} X+\ldots+h_{m-1} X^{m-1}+h X^{m}}{\operatorname{lcm}\left(q_{1}, q_{2}\right)}=\frac{a}{q} F_{1}(X) \ldots F_{s}(X),
$$

we see that $a$ divides $h$ and $q$ divides $\operatorname{lcm}\left(q_{1}, q_{2}\right)$. On the other hand, by comparing the leading coefficients we find

$$
h=t_{1} \ldots t_{s} a \cdot \frac{\operatorname{lcm}\left(q_{1}, q_{2}\right)}{q} .
$$

Now, since $\left(\operatorname{lcm}\left(q_{1}, q_{2}\right)\right) / q$ is an integer and $\Omega(h / d)<s$, (18) shows that at least one of the $t_{i}$ 's, say $t_{1}$, divides $d$. So we have

$$
\left|t_{1}\right| \leq d
$$

Again we proceed to estimate the resultant $R\left(\bar{g}, F_{1}\right)$. As in Theorem 1 , since $\bar{g}$ and $F_{1}$ are relatively prime, we must have $\left|R\left(\bar{g}, F_{1}\right)\right| \geq 1$. If $F_{1}$ decomposes 
as $F_{1}(X)=t_{1}\left(X-\theta_{1}\right) \ldots\left(X-\theta_{r}\right)$, we have

$$
\left|R\left(\bar{g}, F_{1}\right)\right|=\left|t_{1}\right|^{m} \prod_{1 \leq j \leq r}\left|\bar{g}\left(\theta_{j}\right)\right| .
$$

Using (19) together with $\left|\bar{g}\left(\theta_{j}\right)\right| \leq q_{2}\left|g\left(\theta_{j}\right)\right|$ and $g\left(\theta_{j}\right)=-n_{1} f\left(\theta_{j}\right) / n_{2}$, we find

$$
\left|R\left(\bar{g}, F_{1}\right)\right| \leq d^{m} \frac{q_{2}^{r}\left|n_{1}\right|^{r}}{\left|n_{2}\right|^{r}} \prod_{1 \leq j \leq r}\left|f\left(\theta_{j}\right)\right| .
$$

We now proceed to find the upper bound for $\left|f\left(\theta_{j}\right)\right|$. The equality $n_{1} f\left(\theta_{j}\right)+n_{2} g\left(\theta_{j}\right)=0$ implies

$$
\left(\frac{n_{1} a_{0}}{q_{1}}+\frac{n_{2} b_{0}}{q_{2}}\right)+\ldots+\left(\frac{n_{1} a_{m-1}}{q_{1}}+\frac{n_{2} b_{m-1}}{q_{2}}\right) \theta_{j}^{m-1}+\frac{h}{\operatorname{lcm}\left(q_{1}, q_{2}\right)} \theta_{j}^{m}=0 .
$$

Since (16) allows us to divide by $|h|$, we further have

$$
\left|\theta_{j}\right|^{m} \leq \frac{\operatorname{lcm}\left(q_{1}, q_{2}\right)}{|h|}\left(\frac{\left|n_{1}\right| M(f)}{q_{1}}+\frac{\left|n_{2}\right| M(g)}{q_{2}}\right)\left(1+\left|\theta_{j}\right|+\ldots+\left|\theta_{j}\right|^{m-1}\right) .
$$

Therefore, either $\left|\theta_{j}\right| \leq 1$, or if not, then

$$
\left|\theta_{j}\right|^{m}<\frac{q_{1} q_{2}}{|h|}\left(\frac{\left|n_{1}\right| M(f)}{q_{1}}+\frac{\left|n_{2}\right| M(g)}{q_{2}}\right) \frac{\left|\theta_{j}\right|^{m}}{\left|\theta_{j}\right|-1} .
$$

So in both cases we have

$$
\left|\theta_{j}\right|<1+\frac{q_{1} q_{2}}{|h|}\left(\frac{\left|n_{1}\right| M(f)}{q_{1}}+\frac{\left|n_{2}\right| M(g)}{q_{2}}\right),
$$

and since obviously

$$
\left|f\left(\theta_{j}\right)\right| \leq \frac{M(f)}{q_{1}}\left(1+\left|\theta_{j}\right|+\ldots+\left|\theta_{j}\right|^{m}\right)
$$

we obtain the following upper bound for $\left|f\left(\theta_{j}\right)\right|$ :

$$
\left|f\left(\theta_{j}\right)\right|<\frac{M(f)}{q_{1}} \cdot \frac{\left[1+\frac{q_{1} q_{2}}{|h|}\left(\frac{\left|n_{1}\right| M(f)}{q_{1}}+\frac{\left|n_{2}\right| M(g)}{q_{2}}\right)\right]^{m+1}-1}{\frac{q_{1} q_{2}}{|h|}\left(\frac{\left|n_{1}\right| M(f)}{q_{1}}+\frac{\left|n_{2}\right| M(g)}{q_{2}}\right)} .
$$

It is more convenient to use

$$
\left|f\left(\theta_{j}\right)\right|<|h| M(f) \frac{\left[1+\frac{q_{1} q_{2}}{|h|}\left(\frac{\left|n_{1}\right| M(f)}{q_{1}}+\frac{\left|n_{2}\right| M(g)}{q_{2}}\right)\right]^{m+1}}{q_{1}\left[\left|n_{1}\right| q_{2} M(f)+\left|n_{2}\right| q_{1} M(g)\right]},
$$

which further gives

$$
\left|f\left(\theta_{j}\right)\right|<M(f)\left[1+\frac{q_{1} q_{2}}{|h|}\left(\frac{\left|n_{1}\right| M(f)}{q_{1}}+\frac{\left|n_{2}\right| M(g)}{q_{2}}\right)\right]^{m+1},
$$

since $|h| \leq\left|n_{1}\right| q_{2} M(f)+\left|n_{2}\right| q_{1} M(g)$ and $q_{1} \geq 1$. Therefore by (16) we find

$$
\left|f\left(\theta_{j}\right)\right|<M(f)\left(1+\frac{\left|n_{1}\right| q_{2} M(f)+\left|n_{2}\right| q_{1} M(g)}{\left|n_{2}\right|-\left|n_{1}\right| q_{2} M(f)}\right)^{m+1},
$$


that is,

$$
\left|f\left(\theta_{j}\right)\right|<\left|n_{2}\right|^{m+1} M(f)\left(\frac{1+q_{1} M(g)}{\left|n_{2}\right|-\left|n_{1}\right| q_{2} M(f)}\right)^{m+1} .
$$

Together with (20), (21) yields

$$
\left|R\left(\bar{g}, F_{1}\right)\right|<d^{m}\left[q_{2}\left|n_{1}\right| \cdot\left|n_{2}\right|^{m} M(f)\left(\frac{1+q_{1} M(g)}{\left|n_{2}\right|-\left|n_{1}\right| q_{2} M(f)}\right)^{m+1}\right]^{r}
$$

Let us denote $d^{m} q_{2}\left|n_{1}\right| M(f)\left[1+q_{1} M(g)\right]^{m+1}$ by $\alpha$. We shall prove that

$$
\left[\left|n_{2}\right|-\left|n_{1}\right| q_{2} M(f)\right]^{m+1}>\alpha\left|n_{2}\right|^{m},
$$

which by (22) will contradict the fact that $\left|R\left(\bar{g}, F_{1}\right)\right| \geq 1$.

We search for a suitable $\delta>1$ such that $\left|n_{2}\right|-\left|n_{1}\right| q_{2} M(f)>\left|n_{2}\right| / \delta$, which is equivalent to

$$
\left|n_{2}\right|>\left|n_{1}\right| q_{2} M(f) \frac{\delta}{\delta-1} .
$$

For such a $\delta$ we then require

$$
\left(\frac{\left|n_{2}\right|}{\delta}\right)^{m+1}>\alpha\left|n_{2}\right|^{m}
$$

or equivalently

$$
\left|n_{2}\right|>\alpha \delta^{m+1}
$$

So if we find a $\delta>1$ such that $\alpha \delta^{m+1}>\left|n_{1}\right| q_{2} M(f) \delta /(\delta-1)$, then any $n_{2}$ satisfying (25) will also satisfy (23). Such a $\delta$ should verify

$$
(\delta-1) \delta^{m}>\frac{1}{d^{m}\left[1+q_{1} M(g)\right]^{m+1}} .
$$

Denote $d^{m}\left[1+q_{1} M(g)\right]^{m+1}$ by $w$. One candidate for $\delta$ is $1+1 / w$, since obviously

$$
\frac{1}{w}\left(1+\frac{1}{w}\right)^{m}>\frac{1}{w}
$$

So we have proved that for

$$
\left|n_{2}\right|>\left|n_{1}\right| d^{m} q_{2} M(f)\left(1+q_{1} M(g)+\frac{1}{d^{m}\left[1+q_{1} M(g)\right]^{m}}\right)^{m+1}
$$

we have $\left|R\left(\bar{g}, F_{1}\right)\right|<1$, a contradiction. The proof finishes by noting that $q_{2} M(f) \leq H(f) H(g)$ and $q_{1} M(g) \leq H(f) H(g)$.

REMARKS. 1. Since the sharper bound given by (26) still implies (15) and (16), one can use (26) to rephrase Theorem 2 in terms of $q_{1}, q_{2}, M(f)$ and $M(g)$ instead of $H(f)$ and $H(g)$.

2. Corollary 5 follows immediately from (26).

3 . As in the preceding section, we may also consider the case when the polynomial $n_{1} f(X)+n_{2} g(X)$ has no rational roots. In that case, we see from 
(22) that the same conclusion as in Theorem 3 holds, provided that (26) is replaced by

$$
\left|\frac{n_{2}}{n_{1}}\right|>d^{m / 2} q_{2} M(f)\left(1+q_{1} M(g)+\frac{1}{d^{m / 2}\left[1+q_{1} M(g)\right]^{m}}\right)^{m+1} .
$$

3.2. Proof of Corollary 6 . In this case all that remains is to show that our assumptions force $n_{1} f(X)+n_{2} g(X)$ to be primitive.

Let $\lambda=H(f)\left(1+H(g)+[1+H(g)]^{-m}\right)^{m+1}$. Since $\left|n_{1} a_{m}+n_{2} b_{m}\right|=p$, we have either $n_{2}=\left(p-n_{1} a_{m}\right) / b_{m}$, or $n_{2}=-\left(p+n_{1} a_{m}\right) / b_{m}$.

In the first case we must have $p>n_{1} a_{m}$, otherwise our assumption that $\left|n_{2}\right|>\lambda\left|n_{1}\right|$ would imply $p<n_{1} a_{m}-\lambda\left|n_{1} b_{m}\right|<0$, a contradiction. Thus $\left|n_{2}\right|>\lambda\left|n_{1}\right|$ becomes $\left(p-n_{1} a_{m}\right) /\left|b_{m}\right|>\lambda\left|n_{1}\right|$, which further gives

$$
p>\left|n_{1}\right| \cdot[\lambda-H(f)] \text {. }
$$

Assume now that $p$ divides $n_{1} a_{i}+\frac{p-n_{1} a_{m}}{b_{m}} b_{i}$ for all $i \in\{0, \ldots, m-1\}$, that is, $p$ divides $n_{1}\left(a_{i} b_{m}-a_{m} b_{i}\right)$ for all $i \in\{0, \ldots, m-1\}$. Since

$$
\left|n_{1}\left(a_{i} b_{m}-a_{m} b_{i}\right)\right| \leq 2\left|n_{1}\right| H(f) H(g)<\left|n_{1}\right| \cdot[\lambda-H(f)],
$$

the inequality $(27)$ forces $a_{i} b_{m}=a_{m} b_{i}$ for all $i \in\{0, \ldots, m-1\}$, that is, $b_{m} f(X)=a_{m} g(X)$, a contradiction.

Similarly, in the second case we must have $p>-n_{1} a_{m}$, which also implies (27). Assuming now that $p$ divides $n_{1} a_{i}-\frac{p+n_{1} a_{m}}{b_{m}} b_{i}$ for all $i \in\{0, \ldots, m-1\}$, we will get the same contradiction, which completes the proof.

One may improve Corollary 6 as follows. Let $f(X)=a_{0}+\ldots+a_{m} X^{m}$ and $g(X)=b_{0}+\ldots+b_{m} X^{m} \in \mathbb{Z}[X]$ be two relatively prime polynomials of degree $m$. Assume $n_{1}$ and $n_{2}$ are nonzero integers such that $n_{1} a_{m}+n_{2} b_{m}$ is a prime number $p$ and let $h(X)=n_{1} f(X)+n_{2} g(X)$. For any integer $j$ such that $n_{1} a_{m}+j b_{m} \neq 0$, the polynomials $n_{1} f(X)+j g(X)$ and $g(X)$ are relatively prime of degree $m$, with leading coefficients $n_{1} a_{m}+j b_{m}$ and $b_{m}$ respectively. We obviously have $n_{1} a_{m}+j b_{m}+\left(n_{2}-j\right) b_{m}=p$ and

$$
h(X)=n_{1} f(X)+j g(X)+\left(n_{2}-j\right) g(X) .
$$

Let $K(g)=\left(1+H(g)+[1+H(g)]^{-m}\right)^{m+1}$. Then by Corollary $6, h(X)$ is irreducible over $\mathbb{Z}$ if $\left|n_{2}-j\right|>H\left(n_{1} f+j g\right) K(g)$, or equivalently

$$
\left|p-n_{1} a_{m}-j b_{m}\right|>H\left(n_{1} f+j g\right) \cdot\left|b_{m}\right| \cdot K(g) \text {. }
$$

If $p \leq n_{1} a_{m}+j b_{m}$, we find $p<n_{1} a_{m}+j b_{m}-H\left(n_{1} f+j g\right) \cdot\left|b_{m}\right| \cdot K(g)<0$, a contradiction. Therefore we conclude that $h(X)$ is irreducible over $\mathbb{Z}$ for primes $p$ satisfying

$$
p>\min _{j \neq-n_{1} a_{m} / b_{m}}\left\{n_{1} a_{m}+j b_{m}+H\left(n_{1} f+j g\right) \cdot\left|b_{m}\right| \cdot K(g)\right\} .
$$


Similarly, if $n_{1} a_{m}+n_{2} b_{m}=-p$, then $h(X)$ is irreducible over $\mathbb{Z}$ for

$$
p>\min _{j \neq-n_{1} a_{m} / b_{m}}\left\{-n_{1} a_{m}-j b_{m}+H\left(n_{1} f+j g\right) \cdot\left|b_{m}\right| \cdot K(g)\right\} .
$$

Acknowledgements. The author is grateful to Marian Vâjâitu, Mihai $\mathrm{Cipu}$, Alexandru Zaharescu for useful discussions, and to an anonymous referee for valuable suggestions.

\section{References}

[1] M. Cavachi, On a special case of Hilbert's irreducibility theorem, J. Number Theory 82 (2000), 96-99.

[2] M. Cavachi, M. Vâjâitu and A. Zaharescu, A class of irreducible polynomials, J. Ramanujan Math. Soc. 17 (2002), 161-172.

[3] M. Fried, On Hilbert's irreducibility theorem, J. Number Theory 6 (1974), 211-231.

[4] K. Langmann, Der Hilbertsche Irreduzibilitätssatz und Primzahlfragen, J. Reine Angew. Math. 413 (1991), 213-219.

Institute of Mathematics

of the Romanian Academy

P.O. Box 1-764

RO-70700 Bucureşti, Romania

E-mail: Nicolae.Bonciocat@imar.ro

Received on 16.4.2003

and in revised form on 29.12.2003 